\title{
Using Artificial Intelligence Techniques for Design and Knowledge Discovery in Electromagnetic Nanostructures
}

\author{
Yashar Kiarashinejad, Sajjad Abollahramezani, Mohammadreza Zandehshahvar, \\ Omid Hemmatyar, Hossein Maleki, Muliang Zhu, and Ali Adibi \\ School of Electrical and Computer Engineering \\ Georgia Institute of Technology \\ Atlanta, GA 30332-0250 \\ Phone: (404) 385-2738 \\ Fax: (404) 894-4641 \\ Email: ali.adibi@ece.gatech.edu
}

\begin{abstract}
A new approach based on artificial intelligence (AI) techniques for the design and knowledge discovery in electromagnetic nanostructures will be presented. It is shown that by combining effective dimensionality reduction techniques and combining them with deep-learning and machine-learning algorithms, new insight into the physics of lightmatter interaction is obtained. In addition, the dimensionality reduction approach considerably reduces the computation burden in the design of such nanostructures and even provide new design architectures evolved from the initial structures. In addition to explaining the fundamental properties of this approach, its application in design and knowledge discovery in a series of photonic metastructures, especially a new class of on-demand reconfigurable optical metasurfaces, will be discussed and compared with more conventional approaches.
\end{abstract}

Keywords: Neural networks, Dimensionality reduction, Metasurface, Nanophotonic design, Autoencoder, Deep learning, Machine learning 